\title{
Loneliness And Self Concept Among Psychiatric Patients In Port Said Psychiatric Health Hospital
}

\author{
Gehad M. El-MohammadY', Amal S. Mahmoud ${ }^{2}$, Sonia M. El-Sayad ${ }^{3}$ \\ M.Sc ${ }^{1}$, Professor $^{2}$, Assistant Professor ${ }^{3}$ \\ Psychiatric Nursing and Mental Health Department, \\ Faculty of Nursing, Port Said University.
}

\begin{abstract}
Background: psychiatric patients often experience levels of loneliness and diminished self-concept. Loneliness among people diagnosed with psychiatric diseases can decrease self-concept; therefore. Aim: This study aimed to assess the relationship between loneliness and self -concept among psychiatric patients. Subjects and Methods: a descriptive research design was used; the study was conducted at Port-Said Psychiatric Health Hospital. The study involved 214 psychiatric patients from in-patient units and out-patient clinic, the study data were collected by using of the following tools; the Tennessee Self-Concept Scale and University of California, Los Angeles Loneliness Scale. In addition, personal and clinical data questionnaire was added; the data was collected through an interview questionnaire. Results: Study results revealed that, the highest percentage of the studied psychiatric patients had mild loneliness and low self-concept. There was a statistically highly significant difference between loneliness and self-concept among psychiatric patients. Conclusion: There was negative correlation between loneliness and self-concept among psychiatric patients. Recommendations: Psychiatric nurse should educate patient how to alleviate loneliness; also how to perceive strengths for elevation of their self-concept.
\end{abstract}

Keywords: Loneliness, self-concept, psychiatric patients. 


\section{INTRODUCTION}

Society views psychiatric patients as damaged, defective and as less socially marketable than the general population. That perception of these peoples as damaged affects their social status and leads to reduces self-concept and may contribute to loneliness (Rokach \& Sha'ked, 2013).

Loneliness is deprivation of social contact or lack of people available or willing to share social and emotional experiences, a state where an individual has the potential to interact with others but is not doing so, and there is a discrepancy between the actual and desired interaction with others (Minocha, Holland, McNulty, Banks\& Palmer, 2015).

In the study of Badcock et al (2015) on people with psychotic disorders found that the rates of loneliness ranging from $74.7 \%$ in people with delusional disorders and up to $93.8 \%$ in those with depressive psychosis. Experimental manipulations of loneliness increase depressive symptomatology, shyness, anxiety, and fear of negative evaluation, and decreases self-concept, social skills, and overall mood (Cacioppo, Cacioppo \& Boomsm, 2013).

Self-concept is the totality of the individual's thoughts and feelings with reference to himself as an object (Kucuker \&Tekinarslan, 2015). It is one of the most analyzed health-related psychological constructs. It also referred to the perceptions that the individual has about himself, who are based on his experiences with others, interaction with the environment, and the attributions that he makes of his own behavior (García et al, 2014).

The nurse uses many types of interventions to relive loneliness and these interventions categorized into two main groups: group intervention and individual intervention (Brownie\& Horstmanshof, 2011), group intervention methods it is supportive interventions that fall within group services include day centre-type services (such as lunch clubs), and social group schemes which aim to help people widen their social circles (Barnett et al., 2011; Ahmed , 2015), individual intervention methods include: befriending, mentoring, and gatekeeping such as community navigator or way finder initiatives (National Institute for Health Research, 2014).

\section{Significance of the study:}

Loneliness and mental health can become a vicious circle of negative thoughts and emotions. Being socially isolated can be a big factor in loneliness which in turn leads to depression. However, once 
depressed, feelings of anxiety and low self-concept can lead to people removing themselves from their circle of friendships due to perceived stigma about their condition (public health services, 2016).

Loneliness is a painful universal phenomenon it might be a threat on person`s social relations and communication studying the concept of loneliness among psychiatric patients is of a great importance, due to its negative effects on maintaining a healthy personal and social life. It has negatively significant effects on the patients as a general and on their perception of self specifically (Mushtaq, Shoib, Tabindah \& Sahil, 2014).

More than half of people suffering from mental illness are lonely and very little research has focused on the psychiatric patient's experience of loneliness (Coetzee, 2015). So this study aimed to assess the relationship between loneliness and self- concept among psychiatric patients and hope to stimulate more research into the phenomenon of loneliness specifically in the psychiatric perspective.

\section{AIM OF THE STUDY:}

Assess the relationship between loneliness and self-concept among psychiatric patients in Port Said Psychiatric Health Hospital through the following Research objectives:

1. Assess level of loneliness among psychiatric patients.

2. Identify level of self-concept among psychiatric patients.

3. Determine the relationship between loneliness and self-concept among psychiatric patients.

\section{Research question}

Does the experience of loneliness affect self- concept negatively?

\section{SUBJECT AND METHODS:}

\section{Research design:}

A descriptive design was used. 


\section{Setting:}

The present study was carried out at Port Said Psychiatric Health Hospital (PSPHH) including inpatient units and outpatient clinic.

\section{Subjects}

The total sample size estimated for this study was 214 psychiatric patients admitted to the inpatient units and patients came for follow up in the outpatient clinic in Port-Said Psychiatric Health Hospital. Exclusion criteria were patients with brain diseases, mental retardation, acute psychosis and drug addicted patients.

\section{Sample Size:}

The sample size was determined by using the following equation (Dobson, 1984):

$$
\mathbf{Z}^{2}
$$

Sample size $(\mathbf{n})=$

$\Delta^{2}$

This substituting of the equation: $\mathrm{n}=$ sample size, $\mathrm{p}=$ prevalence of loneliness was $85.5 \%$ in people with depressive psychosis (Badcock et al., 2015), $\mathrm{Z}=$ a percentile of the standard normal distribution by $95 \%$ confidence level $=1.96, \Delta^{2}=$ the width of the confidence interval $=5.0$

The calculated sample size is 195 patients. Due to the expected non-participating rate (10\%), the final sample size will be 214 patients with loneliness.

\section{TOOLS:}

\section{Tools of data collection:}

The First tool: Tennessee Self-Concept Scale (second edition): It was developed by (Fitts, 1965), modified by (Fitts \& Michell, 1985) secondary to (Marsh \&Richards, 1987) and translated to Arabic language by (Mohammed, 2008). It includes 100 items measure the different dimensions of self-concept, the participant responded to self-descriptive statements using a 5-point likert scale; from 5-1 for positive statements severity/frequency ranged from: strongly agree, agree, neutral, 
disagree and strongly disagree, score less than $36 \%$ indicated very low self-concept, score from $36 \%-52 \%$ indicated low self- concept, score from 52.1-68\% indicated medium self- concept and score more than $68 \%$ indicated high self- concept.

The second tool: University of California, Los Angeles Loneliness Scale (Version 3): It was developed by Russell (1996) and translated to Arabic language by BenDhnon \& Mohi (2014) in order to assess subjective feelings of loneliness. Using 20 descriptive statements, a score from 1-4 for positive statements severity/frequency ranged from never, rarely, sometimes and always. The higher scores indicated greater degrees of loneliness, the degree of loneliness was categorized according to the following scores; 20-39 scores indicate mild loneliness, 40-59 is moderate and 6080 indicate severe loneliness.

In addition, personal and clinical data questionnaire was added. Personal and clinical characteristic questionnaire developed by the researcher after review of literature. It included personal data such as patient's age, sex, marital status, educational level and occupation and residence. As regarding clinical characteristics, these included outpatient clinic or inpatient, clinical diagnosis, onset of illness, duration of illness, number of previous hospitalizations and duration of current hospitalization ....etc

\section{Pilot Study:}

A pilot study was carried on $10 \%$ (22 psychiatric patients) of the total sample to test the applicability and clarify the feasibility of tools and to estimate the proper time required for answering the questionnaire.

\section{Ethical Considerations:}

A written consent was taken from patients, after explaining the purpose and the importance of the research study. Patients assured about the confidentiality of the information gathered and that it will be used only for the purpose of the study.

\section{Field Work:}

The study subjects comprised of a convenience sample with total number of 214 psychiatric patients who were admitted to the inpatient units and patients came for follow up in the outpatient clinic in the previously mentioned hospital. According to inclusion criteria such as adult patient 
representing different psychiatric disorders of both sexes felt with loneliness, have deficit in selfconcept and didn't have mental illness, diagnosed with neurological problems or inability to communicate.

The data collection took a period of approximately four months and fifteen days started from $20^{\text {th }}$ of august 2016 to $5^{\text {th }}$ January 2017. The study was conducted using the interview technique that was conducted on an individual basis; each patient was interviewed individually for one session. The researcher started to collect data 3 days per week from 9 A.M to 2 P.M. A number of 5-6 patients were interviewed per day. Each interview lasted for 45-60 minutes. The researcher asked patient the needed information then filled in the questionnaire.

\section{STATISTICAL DESIGN:}

Data were coded and transferred into specially designed formats for data entry then data were analyzed and computed. The collected data were organized, categorized, tabulated in tables using numbers and percentage, mean and standard deviation. Chi-square $\left(\mathrm{x}^{2}\right)$ test was used to test the associations among the under studied qualitative variables, the statistical package for social sciences (SPSS version 16.0) was used for statistical analysis. Statistical significance was considered at $\mathrm{p}$-value $<0.05$, and highly significance at $\mathrm{p}$-value $<0.001$

\section{Results:}

The present study was included 214 patients (137 males and 77females) interviewed in in-patient and out- patient departments, their age ranged from 17-69 years with a mean age \& SD of $36.07 \pm 1.3$ years. Regarding their educational level nearly one third of them had a secondary level of education. Regarding working status one third of the studied psychiatric patients reported to work as craftsman and manual worker $(33.7 \%)$, but on the other hand, more than one quarter of the studied psychiatric patients $(29.4 \%)$ were not working. Concerning marital status, more than half of the studied psychiatric patients were single (51.4\%), while, $3.3 \%$ were widowed.

Regarding clinical data of the studied psychiatric patients; the results revealed that nearly two thirds of the studied psychiatric patients $(64.5 \%)$ admitted to in-patient units. It can be also observed that $41.6 \%$ of the studied patients were diagnosed with schizophrenia, while $29 \%$ of them were diagnosed with depression, $21.5 \%$ of them were diagnosed with bipolar disorder. $2.8 \%$ of them were diagnosed with schizoaffective disorder and $5.1 \%$ of them were diagnosed with psychotic 
disorder/ psychosis. Almost half of the studied psychiatric patients (49.3\%) were not satisfied with hospitalization

Table (1): illustrates levels of loneliness as reported by the studied psychiatric patients. As the table shows nearly three quarters of the studied psychiatric patients (74.3\%) had mild loneliness, followed by $20.1 \%$ had moderate loneliness, and $5.6 \%$ of the studied psychiatric patients had sever loneliness. The total means scores and standard deviation of loneliness among the studied psychiatric patients was $(33.21 \pm 12.54)$.

Figure (1): presents levels of self-concept as reported by the studied psychiatric patients. As clear from the table, more than half of the studied psychiatric patients $(53.3 \%)$ had low selfconcept, While, less than one third (31.3\%) had medium self-concept. furthermore, $11.7 \%$ of the studied psychiatric patients had very low self-concept, and finally, 3.7\% had high level of selfconcept.

Table (2): showed dimensions of self-concept as perceived by the studied psychiatric patients. As shown from the table, none of the studied psychiatric patients had high level of moral, family and critical self -concept. On the other hand, about two thirds of psychiatric patients seemed to have low levels of social, personal and physical self-concept $(65.9 \%, 65.4 \%$, and $65.4 \%$ respectively). Finally, the table illustrates that $93.9 \%$ of the studied psychiatric patients had a very low level of critical self-concept

Table (3): illustrates the relationship between levels of self-concept and levels of loneliness as perceived by the studied psychiatric patients, it is clear from the table that, more than three quarters of the studied psychiatric patients who had a very low level of self-concept (83.3\%) had a sever level of loneliness. While, nearly two thirds of the studied psychiatric patients who had low level of self- concept $(65.1 \%)$ had moderate loneliness. Also, more than half of the studied psychiatric patients who had low level of self- concept $(52.8 \%)$ had mild loneliness. Finally, there was a statistically highly significant relation between levels of self-concept and levels of loneliness among the studied patients where $(\mathrm{p}=.000)$.

Table (4): illustrates correlation between total levels of loneliness and total levels of self-concept as perceived by the studied psychiatric patients. As clear from the table a negative correlation was found between levels of loneliness and levels of self-concept $(r=-.507 \& p=0.000)$. 
Table (1): Levels of loneliness as reported by the studied psychiatric patients

\begin{tabular}{|l|l|l||}
\hline Levels of Loneliness & No & $\%$ \\
\hline Mild & $\mathbf{2 1 4}$ & \\
\hline Moderate & 159 & 74.3 \\
\hline Sever & 43 & 20.1 \\
\hline Mean \pm SD & 12 & 5.6 \\
\hline \hline
\end{tabular}

Figure (1): Total levels of self-concept as perceived by the studied psychiatric patients $(n=214)$.

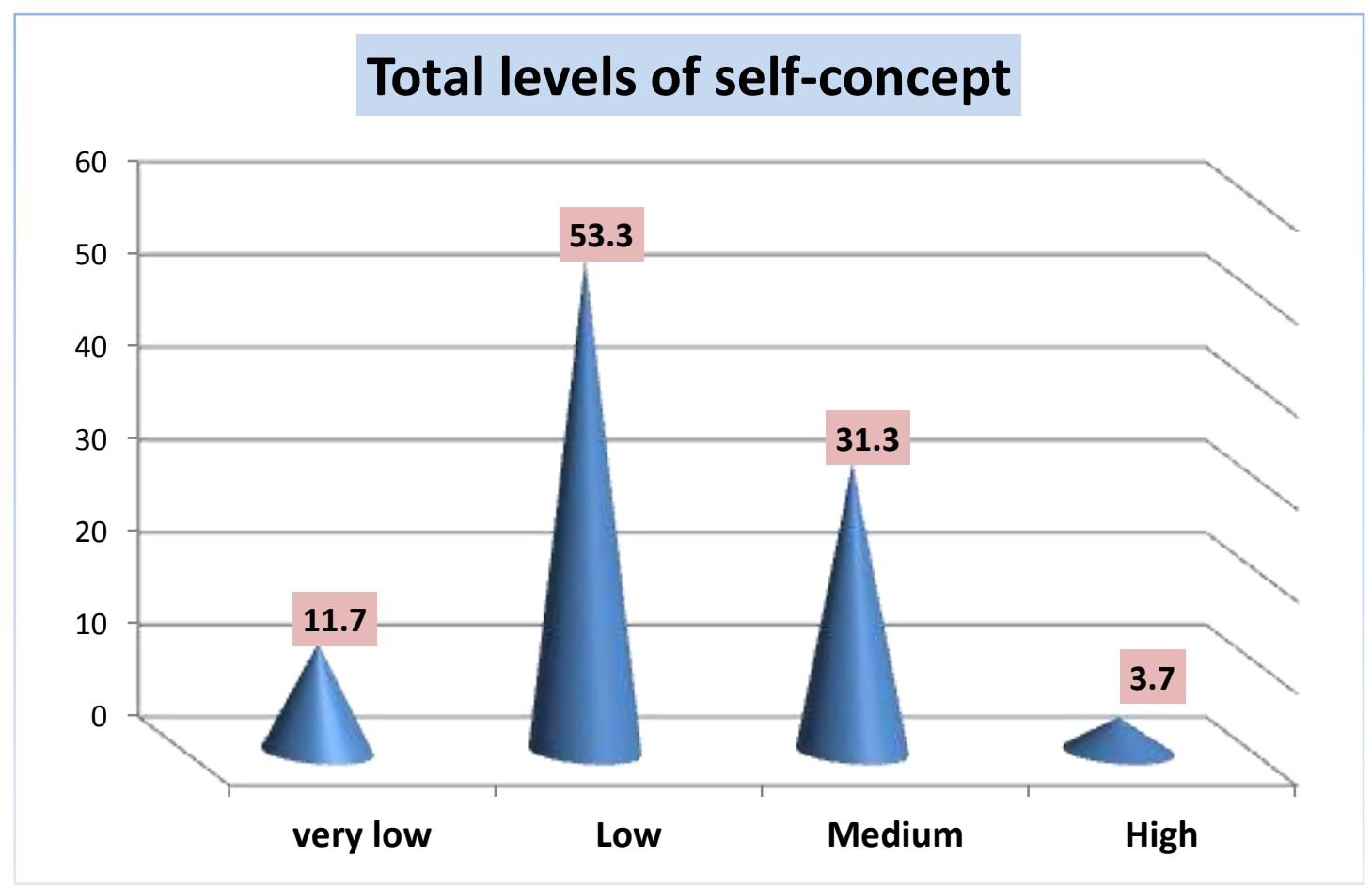


Table (2): Dimensions of self-concept as perceived by the studied psychiatric patients $(n=214)$.

\begin{tabular}{|c|c|c|c|c|c|c|c|c|}
\hline \multirow{3}{*}{ Dimensions } & \multicolumn{8}{|c|}{ Levels of self-concept(n=214) } \\
\hline & \multicolumn{2}{|c|}{ Very low } & \multicolumn{2}{|c|}{ Low } & \multicolumn{2}{|c|}{ Medium } & \multicolumn{2}{|c|}{ High } \\
\hline & No & $\%$ & No & $\%$ & No & $\%$ & No & $\%$ \\
\hline Personal self-concept(@) & 23 & 92.0 & 140 & 65.4 & 49 & 22.9 & 2 & .9 \\
\hline Moral self-concept(@) & 12 & 48.0 & 166 & 77.6 & 36 & 16.8 & 0 & 0.0 \\
\hline Social self-concept(@) & 8 & 32.0 & 141 & 65.9 & 63 & 29.4 & 2 & .9 \\
\hline Family self-concept(@) & 8 & 32.0 & 145 & 67.8 & 61 & 28.5 & 0 & 0.0 \\
\hline Physical self-concept(@) & 9 & 36.0 & 140 & 65.4 & 64 & 29.9 & 1 & .5 \\
\hline critical self-concept(@) & 201 & 93.9 & 13 & 6.1 & 0 & 0.0 & 0 & 0.0 \\
\hline
\end{tabular}

(@) Not mutually exclusive 
Table (3): Relationship between levels of self-concept and levels of loneliness as perceived by the studied psychiatric patients $(\mathrm{n}=214)$.

\begin{tabular}{|c|c|c|c|c|c|c|c|c|c|c|}
\hline \multirow{3}{*}{ Levels of self-concept } & \multicolumn{6}{|c|}{ Levels of loneliness } & & & \multirow{3}{*}{$\begin{array}{l}\chi^{2} \\
\text { Test }\end{array}$} & \multirow{3}{*}{$\begin{array}{l}\text { p- } \\
\text { value }\end{array}$} \\
\hline & \multicolumn{2}{|c|}{$\begin{array}{l}\text { Mild } \\
159\end{array}$} & \multicolumn{2}{|c|}{$\begin{array}{l}\text { Moderate } \\
43\end{array}$} & \multicolumn{2}{|c|}{$\begin{array}{l}\text { Sever } \\
12\end{array}$} & \multicolumn{2}{|c|}{$\begin{array}{l}\text { Total } \\
214\end{array}$} & & \\
\hline & No. & $\%$ & No. & $\%$ & No. & $\%$ & No & $\%$ & & \\
\hline Very low & 5 & 3.2 & 10 & 23.3 & 10 & 83.3 & 25 & 11.7 & & \\
\hline Low & 84 & 52.8 & 28 & 65.1 & 2 & 16.7 & 114 & 53.3 & & .000 \\
\hline Medium & 63 & 39.6 & 4 & 9.3 & 0 & .0 & 67 & 31.3 & & \\
\hline High & 7 & 4.4 & 1 & 2.3 & 0 & .0 & 8 & 3.7 & & \\
\hline
\end{tabular}

$\chi^{2}$ Chi-Square test $\quad *$ significance at $p \leq .05 \quad * *$ highly significance at $p \leq .01$

Table (4): Correlation between total levels of loneliness and total levels of self-concept as perceived by the studied psychiatric patients $(n=214)$.

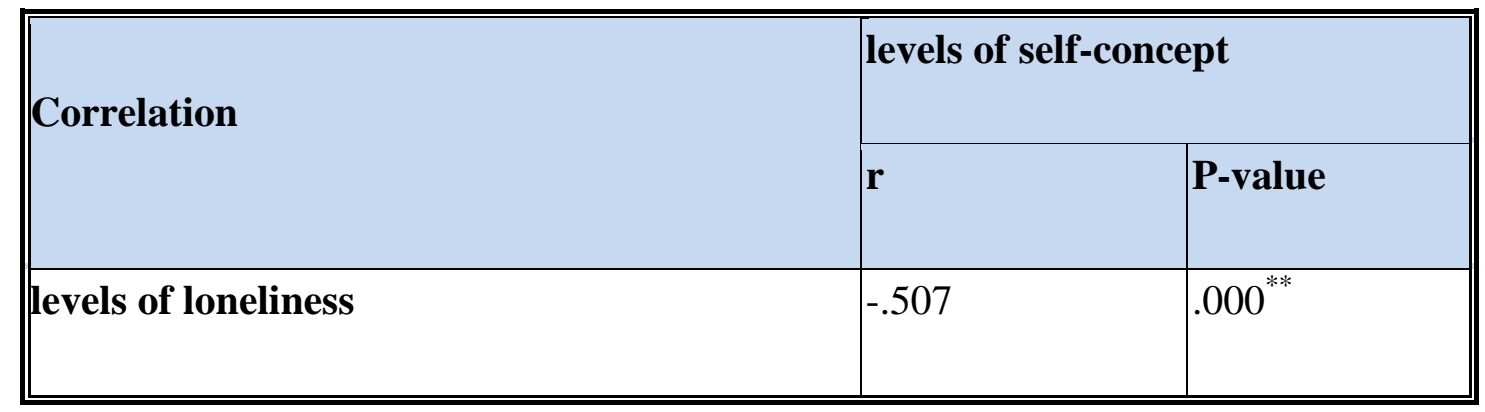

** Correlation is highly significant when $p<0.001$. 


\section{DISCUSSION:}

Loneliness plays an important role in investigating the psychological process of human feelings and behaviors with regard to the formation and maintenance of social relationships (Wu \& Yao, 2008). It is an emotionally unpleasant experience resulting from a discrepancy between the types of interpersonal relationships one wishes to have, and those that one perceives they presently have (Caputo, 2015).

Self-concept through a good social adaptation can provide the power and source for mental health, and poor social adaptation will lead to the hidden trouble about the more psychological problems such as loneliness (Zhu et al., 2016). People with poor mental health are more likely than people with good mental health to feel lonely at all frequency levels (Statistics New Zealand, 2013). Society views psychiatric patients as damaged, defective and as less socially marketable. This perception affects their social status and leads to reduces self-concept and may contribute to loneliness (Rokach \& Sha'ked, 2013).

The present study was conducted to assess the relationship between loneliness and self-concept among psychiatric patients in Port-Said Psychiatric Health Hospital. This study revealed that most of the studied psychiatric patients had mild loneliness. This is may be due to over-control of psychiatric patient's families and their refusal that their patients establish social relationship with others because they consider psychiatric patient a source for stigma to the family. This drives psychiatric patients to sense of inferiority and prefers to be lonely. Emotional loneliness, in which patient can present with others but in the same time, feel alone because lack of support inside hospital, patients complain from health care team who did not listen and care to their problems.

This result was similar to the study of Rizwan \& Ahmad (2015) which done in Karachi, Pakistan and included 260 patients with psychiatric disorders and the study of Tzouvara (2015) which conducted in the Bedfordshire, England, and aimed to investigate the inter-relationships between self-stigma, loneliness, and culture among older adults with mental illness residing in nursing homes. They found that most of the studied patients who had loneliness believe that they are less smart than other people which reinforce isolation. In this way, people with psychiatric illnesses do not trust themselves; they view themselves as awful people who are poor in all aspects of life compared with others. Also, self-stigma and cultural values seem to influence experiences of emotional loneliness among this population. 
The results of this study showed that the highest percentages of patients`scores appeared in relation to lower ratings of all areas of self-concept (physical, moral, personal, family, social and critical) and more than half of all studied patients had low level of overall self-concept. This finding may be due to psychiatric patient's failure to achieve lots of life requirements, for example most of them had low educational level, not satisfying work; patients may not attend their responsibilities and rules in society. Moreover, readmission to psychiatric health hospital may contribute to lack of selfconfidence and increased level of self -stigma; patients may be also unable to meet their sexual needs (marriage) or to success in their marital life because they may be irresponsible or act maladaptive behaviors with partners or other people. All of the above may be explanations for the lower rating of all areas of self-concept.

These results are agreed with Horvat (2015) who carried out a study on Slovenia to examine, how different aspects of self-concept are connected with identity in people with schizophrenia and found that participants with schizophrenia had lower ratings of all areas of self-concept (physical, moral, personal, family, social) and the total self-concept; schizophrenic patients indicated problems in defining the self and indicate a sense of alienation from or disappointment in their families and a lack of social skills.

The present study displayed that; most of the studied psychiatric patients who have severe loneliness had a very low level of self-concept. This finding may be due to neglect of psychiatric patients in their families and lack of community acceptance to deal with the psychiatric patients. This is a realistic result, of course patients who feels alone do not participate with others may feel inadequacy; moreover, the researcher observed that patients tend to compare their lives and social roles before their hospitalization and after hospitalization. These factors in addition to patients complained from not enough recreational activities in the hospital may contribute to low selfconcept as a consequence for feelings of loneliness.

These results are agreed to the result of Shiod et al. (2016) who examined loneliness among people diagnosed with schizophrenia living in Japanese communities, and found that lower level of selfconcept is related to a higher level of loneliness because decreased ability to complete the activities performed before the onset of illness compels individuals to evaluate themselves negatively, which means that they cannot make correct judgments about their ability or strength, leading to greater loneliness, the degree of social support from family members or friends has an effect on loneliness and the higher level of community integration could reduce a level of loneliness; but this results 
was not supported by the result of the study of Schwartz \& Gronemann (2009) which conducted in Israel among a sample of 97 individuals (over age 18) who were diagnosed with schizophrenia ;and did not indicate the internal resource of self-efficacy beliefs as a predictor of loneliness.

The study also displayed that a negative correlation was found between levels of loneliness and levels of self-concept among psychiatric patients. This finding may be due to psychiatric patient's felt with dissatisfaction about all aspects of their life and felt that they are refused from others related to their maladaptive behaviors and stranger thoughts, all of these contribute to reduce their self-concept and increase level of loneliness. Also, self-concept contain different dimensions interacted and related as family, social, physical, personal, moral and neutral as revealed by study. This result was similar to Tharayil (2006) which showed that there is a significant positive relationship between the negative self-concept among participants and their loneliness; stated that unhealthy thoughts and feelings of uselessness and worthless which might lead them to social withdrawal resulting in loneliness.

\section{CONCLUSION:}

Based on the results of this study it can be concluded that; there was negative correlation between loneliness and self-concept among psychiatric patients. If we intervene to manage loneliness, lead to promote self-concept and this may prevent the development of psychiatric disorders.

\section{RECOMMENDATIONS:}

- Psychiatric nurse should improve the perceptions of strengths of the psychiatric patients and assist them to share with continuous community activities to take a more assertive role to elevate their self-concept.

- Assess the social network and relationships of psychiatric patients and educate them how to alleviate loneliness via group intervention such as group therapy and individual intervention such as befriending, mentoring, and gate keeping such as Community Navigator or Way finder initiatives. 


\section{REFERENCES:}

Ahmed, T. (2015). Factors affecting loneliness among instituliatized and non instituliatized, unpublished thesis, Faculty of Nursing, Mansoura University: Egypt.

Badcock, J., Shah, S., Mackinnon, A., Stain, H., Galletly, C., $\quad$ Jablensky, A., \& Morgan, V. (2015). Loneliness in psychotic disorders and its association with cognitive function and symptom profile. Atlas of Science: another view on science: Retrieved from http://atlasofscience.org. Access at 10/2/2016.

Barnett, A., Cann, P., Jopling, K., Victor, C., Scharf, T., Burholt, V., Cattan, M. (2011). Safeguarding the convoy: a call to action from the campaign to end loneliness, Age Uk Oxfordshire: OX14 5BW.

Ben-Dhnon, S. \&Mohi, I. (2014). A sense of loneliness and its relationship with self-esteem among university students, Journal of Humanities and Social Sciences, (16):69-86.

Brownie, S., \& Horstmanshof, L. (2011). The management of loneliness in aged care residents: An Important Therapeutic target for geronotological nursing, Elsevierinc, 32(5), 318-325.

Cacioppo, J., Cacioppo, S., \& Boomsma, D. (2013).Evolutionary mechanisms for loneliness, Cognition \& Emotion available at http://dx.doi.org/10.1080/02699931.2013.837379, access at 22/5/2016.

Caputo, A. (2015). The Relationship between Gratitude and Loneliness: The Potential Benefits of Gratitude for Promoting Social Bonds, Europe's Journal of Psychology, 11(2), 323334.doi:10.5964/ejop.v11i2.826.

Coetzee, J. (2015). Meaning of loneliness: subjective experience of psychiatric patients, published Doctoral thesis in psychiatry, faculty of health sciences, university of Pretoria, South Africa. 
Fitts, H. (1965).Manual for Tennessee self- concept scale, Los Angeles: Western Psychological Services.

García, P., Marcos, L., Guillamón, A., García-Cantó, E., Pérez-Soto, J., Casas, A.,... Lopez,P. (2014). Physical fitness level and its relationship with self-concept in school children. Psychology, (5), 2009-17.

Horvat, B. (2015). Identity in People with Schizophrenia and in those after Spinal Cord Injury. Journal of Psychology and Clinical Psychiatry, 2(5). doi: 10.15406/jpcpy.2015.02.00090.

Kucuker, S., \&Tekinarslan, I. (2015). Comparison of the self-concept, social skills, problem behaviors, and loneliness levels of students with special needs in inclusive classrooms, educational sciences theory \&practice, EDAM, 15(6), 1-16. doi: 10.12738/estp.2015.6.2331.

Marsh, W. \& Richards, E. (1987). The Tennessee self-concept scale: reliability, internal structure, and construct validity, Reports - Research/Technical, (143).

Minocha, S., Holland, C., McNulty, C., Banks, D., \& Palmer, J.(2015).Social isolation and loneliness in people aged 55 and over in Milton Keynes. The Open University, UK: 10115.

Mohamed, S. (2008). Trends kindergarten teachers to work with the child in the light of some psychological and demographic variables, published master thesis, faculty of kindergarten, Cairo University, Egypt.

Mushtaq, R., Shoib, S., Tabindah, S., \& Sahil, M. (2014). Relationship between loneliness, psychiatric disorders and physical health: A review on the psychological aspects of loneliness, Journal of Clinical and Diagnostic Research, 8(9), WE01-WE04.

National Institute for Health Research. (2014).Interventions for loneliness and social isolation, Centre for Reviews and Dissemination, university of York retrieved from 
https://www.york.ac.uk/media/crd/Loneliness\%20and\%20social\%20isolation.pdf access at $1 / 10 / 2016$.

Public health services. (2016). Mental health impact of loneliness \& social isolation, havering, London Borough, retrieved from www.havering.gov.uk/publichealth access at 18/8/2016.

Rizwan, M., \& Ahmad, R.(2015).Self-Esteem Deficits Among Psychiatric, SAGE Open,1-6 DOI: $10.1177 / 2158244015581649$

Rokach, A., \& Sha'ked, A. (2013). Together and Lonely: Loneliness in Intimate RelationshipsCauses and Coping., New York. USA: Nova Science Publishers. ISBN: 978-1-62417-5121

Russell, D. (1996). UCLA Loneliness Scale (Version 3): Reliability, validity, and factor, structure. Journal Of Personality Assessment, (66), 20-40.

Schwartz, C\& Gronemann, O. (2009). The Contribution of Self-efficacy, Social Support and Participation in the Community to Predicting Loneliness among Persons with Schizophrenia Living in Supported Residences, Isr J Psychiatry Relat Sci 46 (2) ,120-129.

Shiod, A., Tadaka, E., \& Okochi, A. (2016).Loneliness and related factors among people with schizophrenia in Japan: a cross-sectional study. Journal of Psychiatric and Mental Health Nursing, (23), 399-408.

Statistics New Zealand. (2013). Loneliness in New Zealand: Findings from the 2010 NZ General Social Survey. Retrieved from www.stats.govt.nz. Access at 15/8/2017.

Tharayil, D. (2006). Self, Social, and Family Perceptions in Relation to Loneliness Controlling for Depression among Individuals with Schizophrenia. The Internet Journal of Mental Health, 4 (1). 
Tzouvara, V.(2015). Self-Stigma, Loneliness and Culture among Older Adults with Mental Illness Residing in Nursing Homes, published doctorate thesis, University of Bedfordshire, England.

Wu, C., \& Yao, G. (2008). Psychometric analysis of the short-form UCLA loneliness scale (ULS-8) in Taiwanese undergraduate students. Personality and Individual Differences,(44),17621771.

Zhu, J., Wang, X., Liu, Z., Liu, T., Wei, G., \&Chen, X. (2016). The Relationship between SelfConcept and Mental Health among Chinese College Students: The Mediating Effect of Social Adjusting. Open Journal of Social Sciences (4). 118-125. ISSN Online: 2327-5960. 
الثعور بالوحدة ومفهوم الذات لاى المرضى النفسيين في مستثفى الصحة النفسية ببورسعيد

جهاد محمد المحمدي، أ.د/ أمل صبحي محمود، أ.م.د/سونيا محمد الصياد

قسم التمريض النفسي و الصحة العقلية.- كلية التمريض - جامعة بورسعيد

\section{الخلاصة}

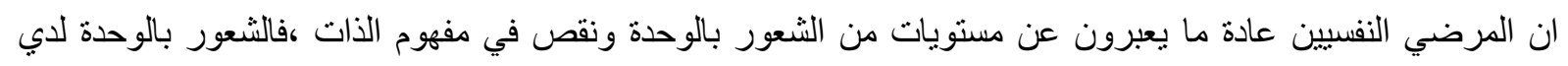

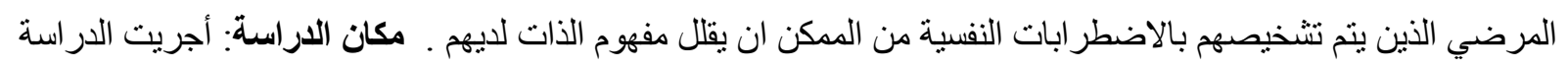

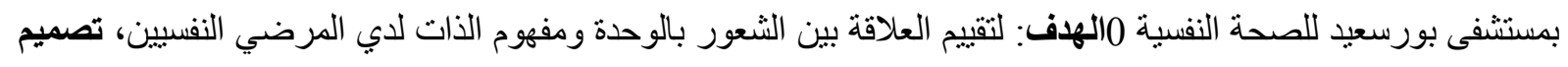
الاراسة: استخدم تصميم وصفي في هذه الدراسة. العينة :تضمنت 214 مريض نفسي من الذين كانوا يتابعون بقسم العيادات

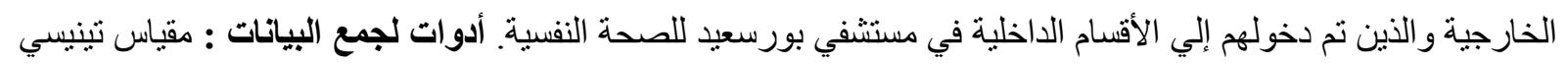

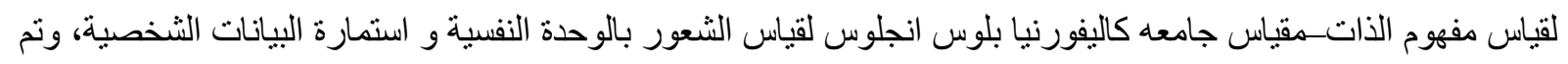

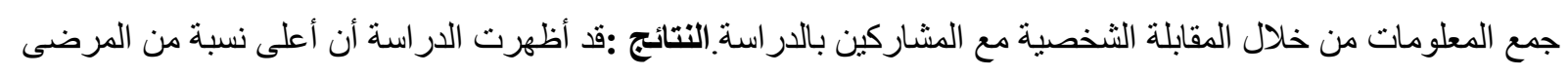

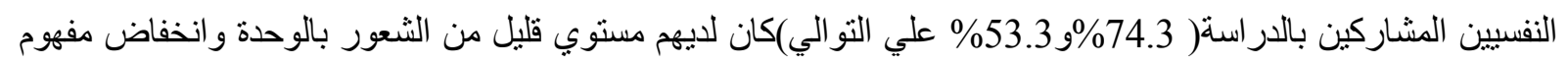

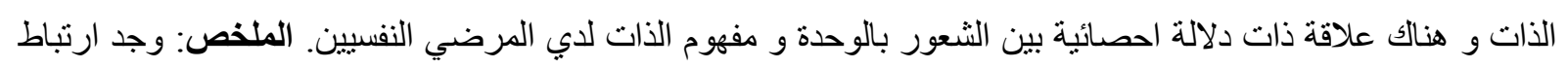

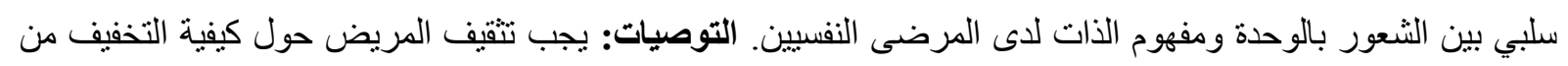

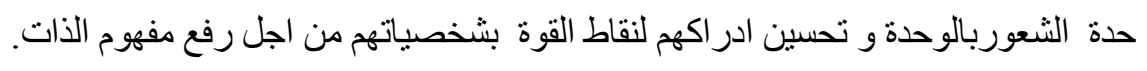

الكلمات المرشدة: الثتعور بالوحدة، مفهوم الذات ، المرضى النفسيين 